\title{
Mining the Dark Web: A Novel Approach for Placing a Dark Website under Investigation
}

\author{
Bassel Alkhatib \\ Syrian Virtual University, Damascus, Syria \\ Al-Sham Private University, Damascus, Syria \\ Email: t_balkhatib@svuonline.org, b.alkhatib.foit@aspu.edu.sy \\ Randa S. Basheer \\ Syrian Virtual University, Damascus, Syria \\ Email: randa_82880@svuonline.org
}

Received: 15 July 2019; Accepted: 11 September 2019; Published: 08 October 2019

\begin{abstract}
In the last two decades, illicit activities have dramatically increased on the Dark Web. Every year, Dark Web witnesses establishing new markets, in which administrators, vendors, and consumers aim to illegal acquisition and consumption. On the other hand, this rapid growth makes it quite difficult for law and security agencies to detect and investigate all those activities with manual analyses. In this paper, we introduce our approach of utilizing data mining techniques to produce useful patterns from a dark web market contents. We start from a brief description of the methodology on which the research stands, then we present the system modules that perform three basic missions: crawling and extracting the entire market data, data pre-processing, and data mining. The data mining methods include generating Association Rules from products' titles, and from the generated rules, we infer conceptual compositions vendors use when promoting their products. Clustering is the second mining aspect, where the system clusters vendors and products. From the generated clusters, we discuss the common characteristics among clustered objects, find the Top Vendors, and analyze products promoted by the latter, in addition to the most viewed and sold items on the market. Overall, this approach helps in placing a dark website under investigation.
\end{abstract}

Index Terms - Dark Web, Dark Web Market, Data Mining, Dark Web Mining, Data Pre-Processing

\section{INTRODUCTION}

Since the rise of the Dark Web in the beginning of 2000s, researches have analyzed activities on the Dark Web using several methodologies and perspectives. Most of these studies focused on terrorists and extremists' groups, though least of the researches considered analyzing dark web markets, where most of the illicit activities and malicious trading take place. Dark Web witnesses launching new markets frequently in many different majors. Products and services on these markets vary in a very wide range, from drugs, weapons, pornography and child abuse, malwares, software exploits and hacking tutorials, renting Botnets for security breaches, in addition to trading documents, fake IDs and stolen credit cards, renting hitmen, and many others. [1]

Researches that analyzed web resources in general considered utilizing data mining and machine learning techniques to explore hidden patterns in massive web data. The discovered hidden patterns lead to new information that helps in understanding different aspects about web resources. In criminology, which is our research area and specifically e-markets in the dark web, data mining plays a significant role in discovering new advantageous information about malicious products promoted in such markets, finding relationships between products and vendors, and the trading size of those vendors. These statistics benefit in inferring whether the vendor is an individual or a company, and the latter may refer to the existence of an organized crime [2]. Furthermore, it helps in understanding the structure of the malicious and criminals' communities in social or psychological views [3]. Information produced by Data Mining methods on dark web markets helps security agencies of different majors in investigations and studying crime and its stewards.

In general, to produce valuable information from a dark web resource, it needs to pass through several stages starting from accessing the dark web, extracting and structuring data, data cleaning and transformation, and finally mining the data.

In the next sections, we present the mining methodology on which our suggested approach depends. The methodology includes a brief description about data mining and web mining, data pre-processing, and the utilized data mining methods. We introduce our approach, which demonstrates a system of three modules: a crawler that accesses a dark web market and extracts data from the entire contents of the market, a cleaner that preprocesses data through cleaning and transforming procedures, and finally a miner. We illustrate in details how the miner analyzes products and vendors' data in 
three basic sides: generating Association Rules from products titles, clustering vendors, and clustering products. Furthermore, we discuss the results gained from the applied methods. This study discusses a method for law and security agencies to put a dark web market under investigation.

\section{RELATED WORK}

Baravalle et al. [2] developed a miner that collects information about products and vendors on Agora market, producing statistics about products ranks according to the deliberated amounts of products, the most trading geographical areas, and names (or nicknames) of their vendors. They stated how such information calls attention to the type and size of in a dark web market, as the size products may refer to the existence of organized crimes in the market.

Robertson et al. [4] discussed supervised and semisupervised approaches in studying dark web markets, by determining the qualitative areas of the products exclusively promoted by a specific vendor. The approach uses manual labeling in addition to clustering techniques to group products in separated categories, utilizes $\mathrm{K}$ Means algorithm to generate centroids and clusters, and then evaluates generated clusters using measures like Rand-index and Entropy.

Quan Le et al. [5] introduced an approach of Malware classification for non-experts. They discussed the importance of Machine Leaning techniques in reducing the manual effort and the time it consumes. The approach starts from data pre-processing, classifies elements into benign and malicious software, and classifies the latter into types according to a previously defined labeling using CNN algorithm.

Celestini et al. [6] introduced an approach for crawling and processing data from markets on the dark web. They discussed drugs trading on the dark web, like AlphaBay, and the importance of monitoring activities on cryptomarkets to produce early warnings on how drugs' trading evolves around the world. The study discussed data preprocessing techniques and preparing data for further analysis.

Marin et al. [7] discussed an approach of mining communities of malware and exploit vendors, focusing on understanding the vendors and their common characteristics. They used clustering methods and different similarity measures to find correlations among vendors' profiles from several markets, common categories they promote in, and the number of products they have in each category. They discussed how this method helps security agencies in understanding and tracking the hacking-related communities.

Furthermore, researchers studied communities' structures of the dark markets. The studies included analyzing the community and interactivity of its members, and crime and violence such trading lead to [3], in addition to collecting information to conclude the generative mechanisms that enable a market on the dark web to operate and survive [8]. Others discussed the teamwork and social involvement of members of such communities, the discussions addressed the usage of some tools that help in understanding and predicting members' behaviors, and how these markets are vulnerable to disruption [9].

Some studies discussed, from a geographical perspective, the considerable changes in revenues and trading traffic after severe changes happen to the market community, such as arresting a significant vendor or shutting down a market [10]. In additions, studies discussed analyzing geographical trafficking of products and exploring a potential relationship between the virtual world and the physical world, highlighting the role of specific parties from different countries in the international and domestic trafficking [11].

\section{Methodology}

In this section, we briefly describe the basic concepts our approach based on. The concepts include Data Mining, Web Mining, Data and Text Pre-processing, and the employed Data Mining methods, which include Association Rules and Clustering.

\section{A. Data Mining and Web Mining}

Data Mining is the automatic study, which a computer system performs on a huge database in order to extract new hidden relational and statistical information. Data Mining helps in discovering invisible relationships and patterns among raw data elements, and concluding future predictions for new elements, or exploring the common features in order to distribute elements into groups of the same features. Researches also describe Data Mining as Knowledge Discovery in Databases (KDD). [12]

Web Mining on the other hand, is the implementation of data mining techniques on the content, structure and usage of web resources, to identify valid unknown patterns with potential interest from massive amounts of web data. [13]

\section{B. Data and Text Pre-Processing}

Cleaning and preparing data aim to acquire higher efficiency and effectiveness in the mining process. Researches call this stage Pre-processing, and indicate that pre-processing methods can reduce from $50 \%$ to $80 \%$ of the total mining process. [14]

Text pre-processing solves the problem of the feature space high dimensionality, where features (or terms) can exceed tens or hundreds of thousands in count. It also makes text analysis more accurate, and saves time and space [14]

Text enters a series of steps including some or all of the following: $[15,16]$

1. Extraction, which tokenizes the text

2. Lowercase Conversion

3. Special Characters Removal

4. Stopwords Removal

5. Stemming and Lemmatization 
6. Pruning rare words (as they lead to noise in data), using Document Frequency (DF).

7. TF-IDF Weighting

\section{Data Mining Methods}

This section includes brief descriptions of mining methods we used in our approach.

\section{1) Association Rules}

Association Rules Mining is the process of discovering patterns, relationships and information structures inside groups of objects in transactional databases and other information repositories. [17]

The most common use of association rules is in marketing, by analyzing purchasing traffics, which aims to identify groups of products that consumers buy often together, in addition to other fields such as telecommunications, risk management, stock control, agriculture and others. [18]

Generating association rules depends on two important measures: Support and Confidence, by generating all rules that fulfill support and confidence greater than userdefined thresholds named minsup and minconf respectively. [18]

One of the most common and efficient methods of association rules is FP-Tree.

\section{2) Clustering}

Clustering, or Cluster Analysis, is the process of dividing a huge amount of unordered data in a small number of consistent and meaningful groups, by assigning similar elements to one group, which is represented by a center. These groups have three main characteristics: 1) they are relatively homogeneous within themselves, 2) heterogeneous among each other; 3) measuring homogeneity and heterogeneity are according to pre-defined parameters, and using similarity and distance measures, such as Euclidean Distance and Cosine Similarity, to compare the elements to the center. These groups form the clusters, while the centers of the clusters represent the centroids. [19]

In Documents Clustering on the other hand, the process starts by preparing the documents through Feature Extraction, which links each document with a brief representation of its topic using Vector Space Model or Bag of Words Model. The process then calculates centroid vectors, which represent the average weight of documents in the cluster, and measures similarity between two documents using measures like Cosine Similarity that calculates the angle between two vectors. [20]

In our study, we employ two methods of NonHierarchal Clustering, which are K-Means and KMedoids.

\section{SYSTEM ARCHITECTURE}

In this section, we demonstrate the suggested system modules that perform three basic missions: crawling a dark web market, pre-processing extracted data, and mining the processed data. The market under study (which we consider not mentioning for security reasons) uses a $\operatorname{Tor}^{l}$ service as a host, thus it needs a connection to Tor network, in addition to a pre-registration and login. The market is specialized in promoting and selling illicit and malicious products of several different types, distributed over 12 categories.

\section{A. System Components}

Our system consists of three basic modules:

1. The dark crawler Darky

2. The Cleaner

3. The Dark Miner

In the crawling phase, we employ our crawler Darky [21] to access and crawl the website, and extract data from products and vendors' pages. In the second phase, we developed a cleaning module that applies preprocessing techniques to clean and transform data. Finally, we perform data mining methods on clean data using different strategies to extract patterns. These patterns can be quite useful for various agencies in inferring information about products, vendors and their promoting styles. Fig.1 illustrates the three modules:

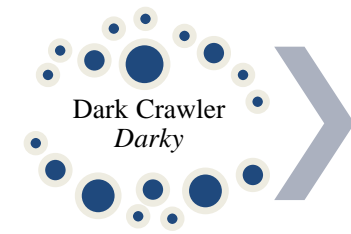

- Setting Connection

- Accessing Website

- Crawling and Data

Extracting

- Saving Data

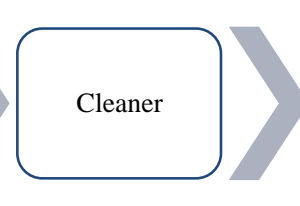

- Reading Files - Cleaning Texts

- Data

Transformation

- File Format

Transformation

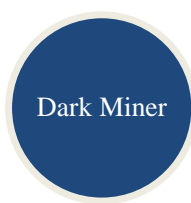

- Association Rules

- Vendors Clustering

- Products Clustering
Fig.1. System Modules

\section{B. Data Collection}

In earlier stage, we developed a dark crawler Darky ${ }^{2}$ using Scrapy ${ }^{3}$. The crawler starts by setting a connection to Tor network through Tor software integrated with Privoxy ${ }^{4}$. After setting the connection, the crawler simulates a user login with credentials we previously created on a dark web market (which we consider not mentioning for security reasons), crawls the entire website pages extracting and directly structuring data without downloading any page, and saving data in separated json files.

The data includes records of 6387 products and 179 vendors shown in Table 1.

\footnotetext{
${ }^{1}$ Tor Project, https://www.torproject.org

${ }^{2}$ Refer to a previous published article for details about Darky and crawling the dark web, available at:

https://doi.org/10.6025/jdim/2019/17/2/51-60

${ }^{3}$ Scrapy, https://scrapy.org

${ }^{4}$ Privoxy, https://www.privoxy.org
} 
Table 1. Number of Vendors and Products Extracted from the Dark Web Market

\begin{tabular}{|c|c|c|}
\hline \multicolumn{3}{|c|}{ Vendors' Total $=179$} \\
\hline & Products Total $=6387$ \\
\hline 1 & Category & Number of Products \\
\hline 2 & Carded Items & 27 \\
\hline 3 & Counterfeit Items & 92 \\
\hline 4 & Digital Products & 2179 \\
\hline 5 & Drugs \& Chemicals & 1569 \\
\hline 6 & Fraud & 887 \\
\hline 7 & Guides \& Tutorials & 987 \\
\hline 8 & Jewels \& Gold & 15 \\
\hline 9 & Other Listings & 243 \\
\hline 10 & Security \& Hosting & 50 \\
\hline 11 & Services & 139 \\
\hline 12 & Software \& Malware & 185 \\
\hline
\end{tabular}

\section{Data Pre-Processing}

Due to the difficulty of reading data in its current format, we transform data into another format that is easy to read and mine. In the pre-processing module, we developed the Cleaner. The main mission of the Cleaner we summarize as follows:

1) Reading elements from json files, and for the element "Title", the Cleaner processes it by removing non-alphabetic (or meaningless) characters, then removing Stopwords

2) Adding the read elements to a new json file

3) Transforming json file to a new file with $x l s x$ format ready for mining

As we previously designed our crawler, here we need two versions of the cleaner as well, one to process products data, and the other to process vendors' data.

Fig. 2 illustrates how the Cleaner works on products data:

The Cleaner processes products data as follows:

1. Starts from a list of files names that contain the products

2. Reads the elements from each file

3. Processes the Title by transforming it to lowercase, then removing the non-alphabetical characters. Using re library, we defined a regular expression that contains all removable characters used in titles. Some of the characters are common (like periods, commas and brackets), and others are rarely used or with strange shapes (like the symbols we noticed vendors often use many of these characters in products titles to draw attention to their products. We excluded some characters that may affect the meaning of the title (like - and \%). Furthermore, the cleaner removes Stopwords, we used nltk.corpus library for this mission, which contains a previously defined list of Stopwords in English.

4. Reads the rest of the element attributes with simple cleaning of some brackets
5. Adds the processed elements to a new data array

6. Moves to the next file

7. Empties the array in a new json file

8. Transforms data from the last json file to a spreadsheet with xlsx format, here we used pandas library

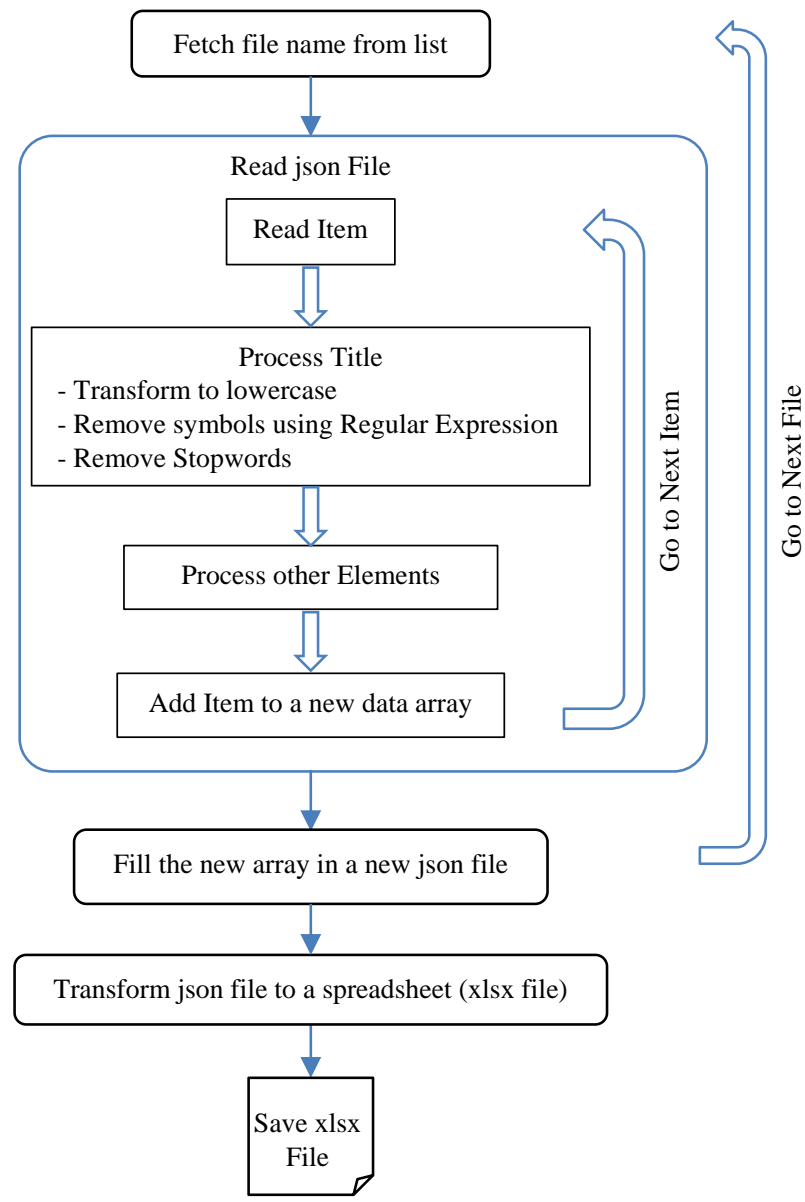

Fig.2. The Cleaner Workflow

With similar but simpler steps, the Cleaner transforms the vendors' data file.

From this stage, we obtained two spreadsheets files: products and vendors.

Table 2 shows examples of products titles before and after processing:

\section{Data Mining Strategies}

In this stage, we used RapidMiner ${ }^{1}$ to design the processes we performed on the extracted and cleaned data, experimenting our methods to study the entire website content.

\section{1) Association Rules Process Design}

As mentioned before, the most common use of association rules is in the field of analyzing shopping traffic. However, we employed this technique in a different purpose, which is analyzing promotion styles. In our approach, we apply the concept of association rules

${ }^{1}$ RapidMiner, https://rapidminer.com/ 
on words, i.e. studying the words that frequently occur together in illicit products titles. This method aims to show the most used words by vendors when they promote their products on the market. We accomplished this approach by considering the "Title" as the transaction and the words as the items.

Table 2. Examples of Products Titles before and after Processing

\begin{tabular}{|c|c|}
\hline Title before processing & Title after processing \\
\hline $\begin{array}{l}\star \text { LSD-25 Blotter } 250 \mu \mathrm{g} \star \text { Qty: } \\
250\end{array}$ & lsd- 25 blotter $250 \mu$ g qty 250 \\
\hline 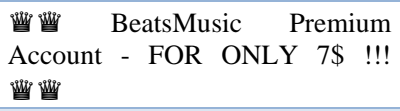 & beatsmusic premium account -7 \\
\hline $\begin{array}{l}\star \star \text { Identity Finder -- Best Data } \\
\text { stealer } \star \star\end{array}$ & identity finder -- best data stealer \\
\hline $\begin{array}{l}\star \text { Ketamine R High Quality } 87 \% \\
\star \quad \text { Full Crystal's } \star € 17.5 \star \quad \text { EC } \\
\text { PUREST LABTEST EVER } \\
97 \% !\end{array}$ & $\begin{array}{l}\text { ketamine } r \text { high quality } 87 \% \text { full } \\
\text { crystal } € 17.5 \text { ec purest labtest } \\
\text { ever } 97 \%\end{array}$ \\
\hline
\end{tabular}

The patterns of the frequently used words may give the analyzer an idea about the most promoted products or the most used combinations of words in titling products. These rules can also help in finding conceptual compositions.

The process represents documents by vectors of words, using TF-IDF to weight words. The process then performs two basic steps: First, it generates the FPGrowth tree to extract frequent words patterns, using Support threshold to determine the desired minimum number of patterns recurrence. Second, it creates association rules from the produced tree using Confidence threshold to determine the desired power of the rule.

Fig.3 illustrates the process design of generating association rules out of products titles, and Fig.4 illustrates the sub-process of documents processing and it consists of a Tokenization operation:

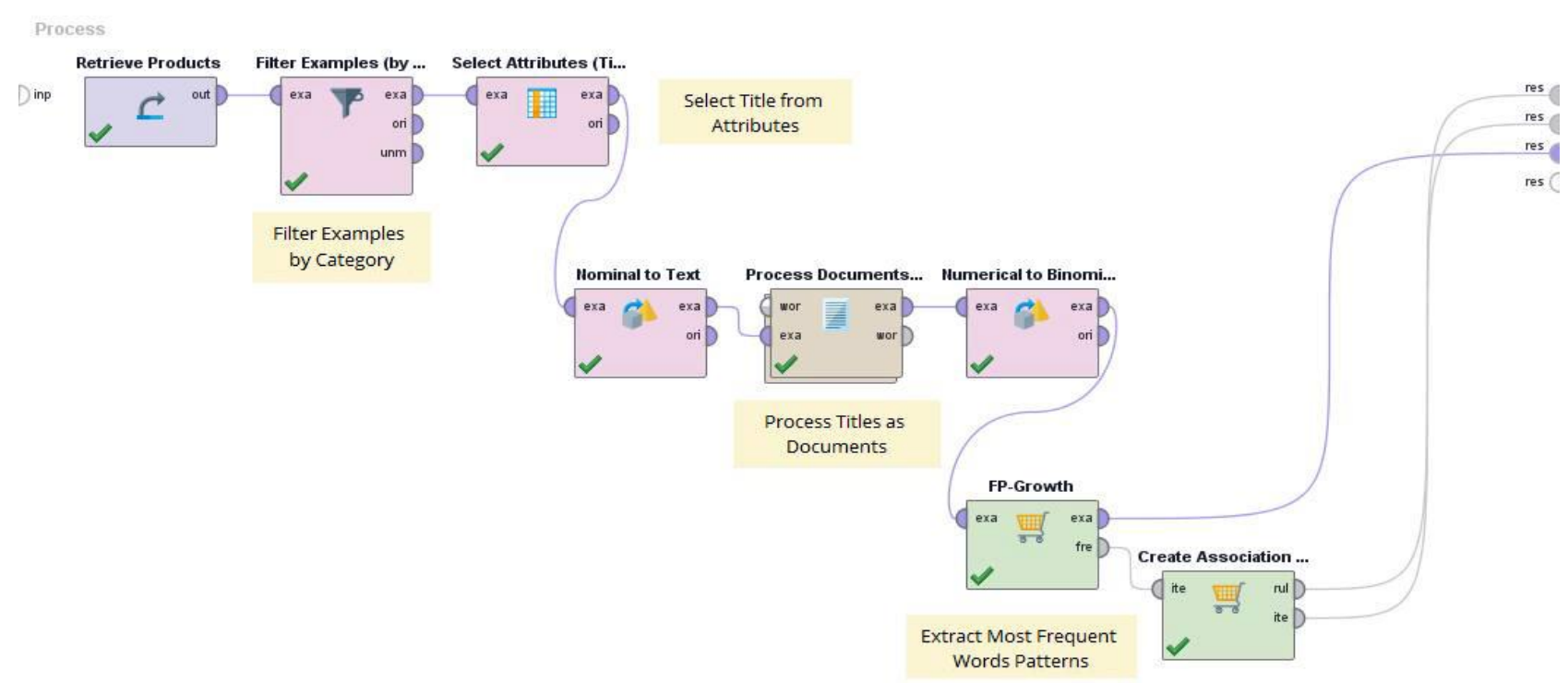

Fig.3. Association Rules Process Design

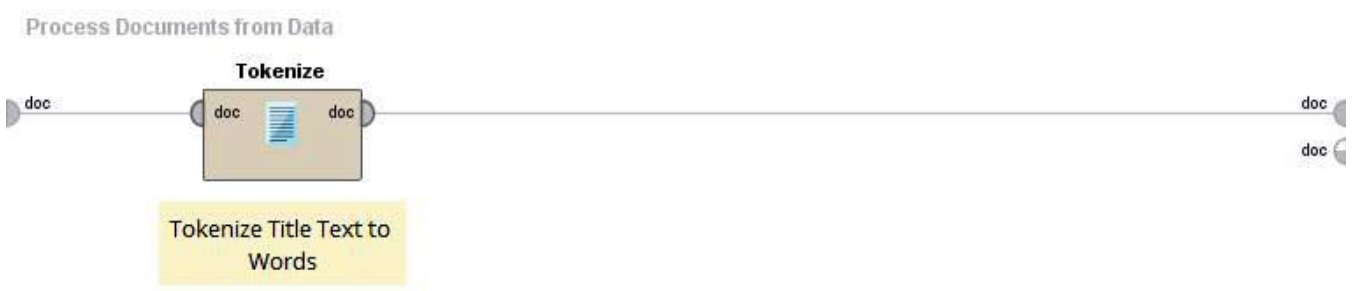

Fig.4. Document Processing Sub-Process

\section{i. Association Rules Results}

The market has products distributed into categories. Therefore, we divide the data to examples according to the corresponding category.
Table 3 shows the number of frequent itemsets and the maximum size of an itemset according to different values of Support and Confidence (for the other categories, they did not show results): 
Table 3. Number of Generated Frequent Itemsets According to Different Support and Confidence Values

\begin{tabular}{|c|c|c|c|c|c|c|c|}
\hline Category & No. of Examples & No. of Items & Sup. & Conf. & No. of Itemsets & Maximum Itemset Size & No. of Rules \\
\hline \multirow{2}{*}{ Counterfeit Items } & \multirow{2}{*}{92} & \multirow{2}{*}{264} & 0.12 & 0.8 & 15 & 4 & 43 \\
\hline & & & 0.13 & 0.85 & 15 & 4 & 43 \\
\hline \multirow{2}{*}{ Digital Products } & \multirow{2}{*}{2179} & \multirow{2}{*}{2038} & 0.2 & 0.65 & 14 & 3 & 13 \\
\hline & & & 0.3 & 0.7 & 5 & 2 & 2 \\
\hline \multirow{2}{*}{ Jewels \& Gold } & \multirow{2}{*}{15} & \multirow{2}{*}{48} & 0.2 & 0.65 & 79 & 6 & 569 \\
\hline & & & 0.3 & 0.7 & 8 & 2 & 3 \\
\hline \multirow{2}{*}{ Other Listings } & \multirow{2}{*}{243} & \multirow{2}{*}{546} & 0.5 & 0.85 & 23 & 4 & 88 \\
\hline & & & 0.55 & 0.95 & 9 & 3 & 10 \\
\hline \multirow{2}{*}{ Security \& Hosting } & \multirow{2}{*}{50} & \multirow{2}{*}{133} & 0.1 & 0.65 & 23 & 4 & 46 \\
\hline & & & 0.2 & 0.7 & 5 & 2 & 1 \\
\hline \multirow{2}{*}{ Services } & \multirow{2}{*}{139} & \multirow{2}{*}{256} & 0.15 & 0.65 & 17 & 4 & 44 \\
\hline & & & 0.2 & 0.75 & 3 & 2 & 1 \\
\hline \multirow{2}{*}{ Software \& Malware } & \multirow{2}{*}{185} & \multirow{2}{*}{331} & 0.15 & 0.7 & 4 & 2 & 1 \\
\hline & & & 0.2 & 0.75 & 4 & 2 & 1 \\
\hline
\end{tabular}

To explain these results, we will take "Counterfeit Items" category. Table 4 shows the generated itemsets from "Counterfeit Items" category with the values 0.13 and 0.85 for Support and Confidence respectively, and sorted by the highest Support, and Table 5 shows examples of association rules generated from these itemsets:

Table 4. Itemsets Generated from "Counterfeit Items" Category

\begin{tabular}{|l|l|l|l|l|l|}
\hline Size & Support & Item 1 & Item 2 & Item 3 & Item 4 \\
\hline 1 & 0.413 & forged & & & \\
\hline 1 & 0.13 & labels & & & \\
\hline 1 & 0.13 & prescription & & & \\
\hline 1 & 0.13 & rx & & & \\
\hline 2 & 0.13 & forged & labels & & \\
\hline 2 & 0.13 & forged & prescription & & \\
\hline 2 & 0.13 & forged & rx & & \\
\hline 2 & 0.13 & labels & prescription & & \\
\hline 2 & 0.13 & labels & rx & & \\
\hline 2 & 0.13 & prescription & rx & & \\
\hline 3 & 0.13 & forged & labels & prescription & \\
\hline 3 & 0.13 & forged & labels & rx & \\
\hline 3 & 0.13 & forged & prescription & rx & \\
\hline 3 & 0.13 & labels & prescription & rx & \\
\hline 4 & 0.13 & forged & labels & prescription & rx \\
\hline
\end{tabular}

Table 5. Examples of Association Rules Generated for "Counterfeit Items" Category

\begin{tabular}{|l|l|}
\hline \multicolumn{1}{|c|}{ Rule } & \multicolumn{1}{c|}{ Confidence } \\
\hline [labels] --> [forged] & (confidence: 1.000$)$ \\
\hline [prescription] --> [forged] & (confidence: 1.000$)$ \\
\hline [labels] --> [forged, prescription] & (confidence: 1.000$)$ \\
\hline [forged, labels] --> [prescription] & (confidence: 1.000$)$ \\
\hline [forged, prescription] --> [labels] & (confidence: 1.000$)$ \\
\hline [forged, labels] --> [rx] & (confidence: 1.000$)$ \\
\hline [prescription] --> [forged, rx] & (confidence: 1.000$)$ \\
\hline [forged, prescription] --> [rx] & (confidence: 1.000$)$ \\
\hline [forged, rx] --> [prescription] & (confidence: 1.000$)$ \\
\hline [prescription, rx] --> [forged] & (confidence: 1.000$)$ \\
\hline$[$ labels, rx] --> [prescription] & (confidence: 1.000$)$ \\
\hline$[$ labels] --> [forged, prescription, rx] & (confidence: 1.000$)$ \\
\hline [forged, labels] --> [prescription, rx] & (confidence: 1.000$)$ \\
\hline [prescription] --> [forged, labels, rx] & (confidence: 1.000$)$ \\
\hline [forged, prescription] --> [labels, rx] & (confidence: 1.000$)$ \\
\hline [labels, prescription] --> [forged, rx] & (confidence: 1.000$)$ \\
\hline [rx] --> [forged, labels, prescription] & (confidence: 1.000$)$ \\
\hline [forged, rx] --> [labels, prescription] & (confidence: 1.000$)$ \\
\hline [forged, labels, rx] --> [prescription] & (confidence: 1.000$)$ \\
\hline
\end{tabular}

Taking a sample word, Fig.5 illustrates association rules related to the element (word) "prescription" with the Support and Confidence of each rule:

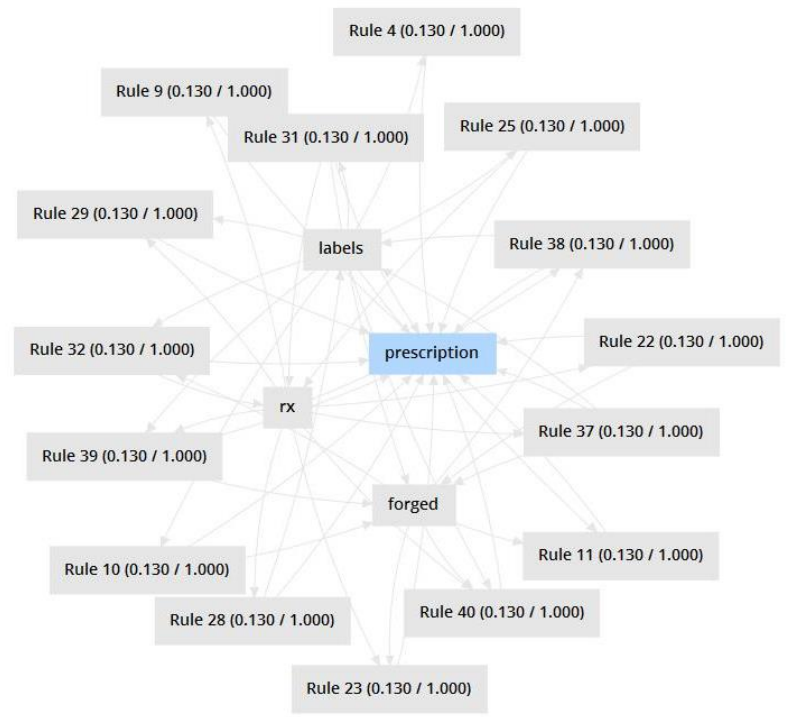

Fig.5. Association Rules Linked with the Word "prescription"

From this approach, we can also conclude the conceptual compositions used when promoting products in each category. Table 6 shows the conceptual compositions resulted from association rules in each category. We choose the compositions with size greater than 2:

\section{2) Clustering Processes Design}

We apply clustering concepts on two aspects: clustering vendors and clustering products.

1. Clustering Vendors

The available data about vendors are the following:

- Vendor Name

- Vendor Level

- Trust Level

- $\quad$ Positive Feedback

- Date of Membership

- Number (presented between two parentheses without any prefix or explanation) 
From the above, we use the three attributes "Vendor Level", "Trust Level", and "Number" to cluster vendors. We chose these attributes due to the distinguished disparity of values we noticed among different vendors, and a potential relationship may exist between the Number and both Vendor Level and Trust Level.

Table 6. The Conceptual Compositions Concluded from the Generating Association Rules from Products Titles

\begin{tabular}{|c|c|}
\hline Category & Conceptual Compositions \\
\hline \multirow{5}{*}{ Counterfeit Items } & forged, labels, prescription \\
\hline & forged, labels, rx \\
\hline & forged, prescription, rx \\
\hline & labels, prescription, rx \\
\hline & forged, labels, prescription, $\mathrm{rx}$ \\
\hline \multirow{2}{*}{ Digital Products } & account, porn, premium \\
\hline & account, premium, warranty \\
\hline \multirow{7}{*}{ Jewels \& Gold } & audemars, royal, oak \\
\hline & audemars, stainless, steel \\
\hline & audemars, piguet, royal, oak \\
\hline & audemars, piguet, stainless, steel \\
\hline & audemars, piguet, oak, stainless, steel \\
\hline & piguet, royal, oak, stainless, steel \\
\hline & audemars, piguet, royal, oak, stainless, steel \\
\hline \multirow{5}{*}{ Other Listings } & account, lifetime, premium \\
\hline & account, lifetime, porn \\
\hline & account, premium, porn \\
\hline & lifetime, premium, porn \\
\hline & account, lifetime, premium, porn \\
\hline \multirow{6}{*}{$\begin{array}{c}\text { Security \& } \\
\text { Hosting }\end{array}$} & vpn, account, premium \\
\hline & premium, warranty, lifetime \\
\hline & premium, warranty, accounts \\
\hline & premium, lifetime, accounts \\
\hline & warranty, lifetime, accounts \\
\hline & premium, warranty, lifetime, accounts \\
\hline \multirow{5}{*}{ Services } & famous, get, way \\
\hline & famous, get, easy \\
\hline & famous, way, easy \\
\hline & get, way, easy \\
\hline & famous, get, way, easy \\
\hline
\end{tabular}

Before starting the clustering process, we apply a performance evaluation to help in choosing the suitable clustering algorithm and the number of clusters to generate. Fig.6 illustrates the steps of performance evaluation process for both K-Means and K-Medoids using Davies-Bouldin Index ${ }^{1}$, with different numbers of clusters and the Euclidean Distance measure.

Table 7 shows the resultant values of Davies-Bouldin Index in performance evaluation of both algorithms:

\footnotetext{
${ }^{1}$ Davies-Bouldin Index factor stands on the principle that "the algorithm which produces clusters with minimum intra-distances, and maximum inter-distances will have minimum value of Davies-Bouldin Index", i.e. it is the percentage of internal distances of clusters elements on external distances of clusters. Therefore, the smaller its value, the better the clustering is. [22]
}

Table 7. Davies-Bouldin Index Values for both K-Means and KMedoids Algorithms in Vendors' Clustering with Different Numbers of Clusters

\begin{tabular}{|c|c|c|}
\hline Number of Clusters & K-Means & K-Medoids \\
\hline 2 & 0.310 & 0.275 \\
\hline 3 & 0.389 & 0.376 \\
\hline 4 & 0.312 & 0.493 \\
\hline 5 & 0.387 & 0.798 \\
\hline 6 & 0.364 & 0.499 \\
\hline
\end{tabular}

From this table we can see that clustering using KMedoids with $\mathrm{k}=2$ gives the least value of the index thus the best clustering. Relying on this result, we design the vendors' clustering process illustrated in Fig.7. In the end of this process, it stores Top Vendors' names for later use (explained in the next section):

\section{i. $\quad$ Clustering Vendors Results}

By completing the above process, we have the following distribution of elements on clusters:

\section{Cluster Model:}

Cluster 0: 172 items

Cluster 1: 7 items

Total number of items: 179

Table 8 includes values of the clusters centroids, and Table 9 shows Top Vendors' data in "cluster_1". According to the three attributes, we notice relatively high values of the Number against values of the Vendor Level and Trust Level, which they range between 2 and 5 . This number may refer to the level of interactivity or professionalism of the vendors inside the market community; therefore, we considered them as Top Vendors:

Table 8. Centroids Values in the Generated Clusters of Vendors

\begin{tabular}{|c|c|c|}
\hline Attribute & cluster_0 & cluster_1 \\
\hline number & 0 & 1243 \\
\hline tlevel & 1 & 4 \\
\hline vlevel & 1 & 5 \\
\hline
\end{tabular}

Table 9. Top Vendors' Data

\begin{tabular}{|c|l|c|c|c|c|c|c|}
\hline \multicolumn{1}{|c|}{ name } & \multicolumn{1}{c|}{ cluster } & number & tlevel & vlevel & member & positive \\
\hline Lepricon & cluster_1 & 1045 & 2 & 3 & $\begin{array}{c}\text { Fri Mar } \\
162018\end{array}$ & 95.77 \\
\hline Underworld & cluster_1 & 742 & 3 & 4 & $\begin{array}{c}\text { Sat Apr } \\
072018\end{array}$ & 92.61 \\
\hline DrunkDragon & cluster_1 & 1919 & 1 & 2 & $\begin{array}{c}\text { Sat Feb } \\
032018\end{array}$ & 93.06 \\
\hline rvaska & cluster_1 & 1316 & 1 & 2 & $\begin{array}{c}\text { Thu Feb } \\
222018\end{array}$ & 95.71 \\
\hline GodsLeftNut & cluster_1 & 1022 & 1 & 2 & $\begin{array}{c}\text { Fri Apr } \\
202018\end{array}$ & 97.98 \\
\hline TikTokCC & cluster_1 & 901 & 3 & 4 & $\begin{array}{c}\text { Wed Apr } \\
182018\end{array}$ & 98.15 \\
\hline savastano & cluster_1 & 1243 & 4 & 5 & $\begin{array}{c}\text { Mon Apr } \\
162018\end{array}$ & 98.75 \\
\hline
\end{tabular}




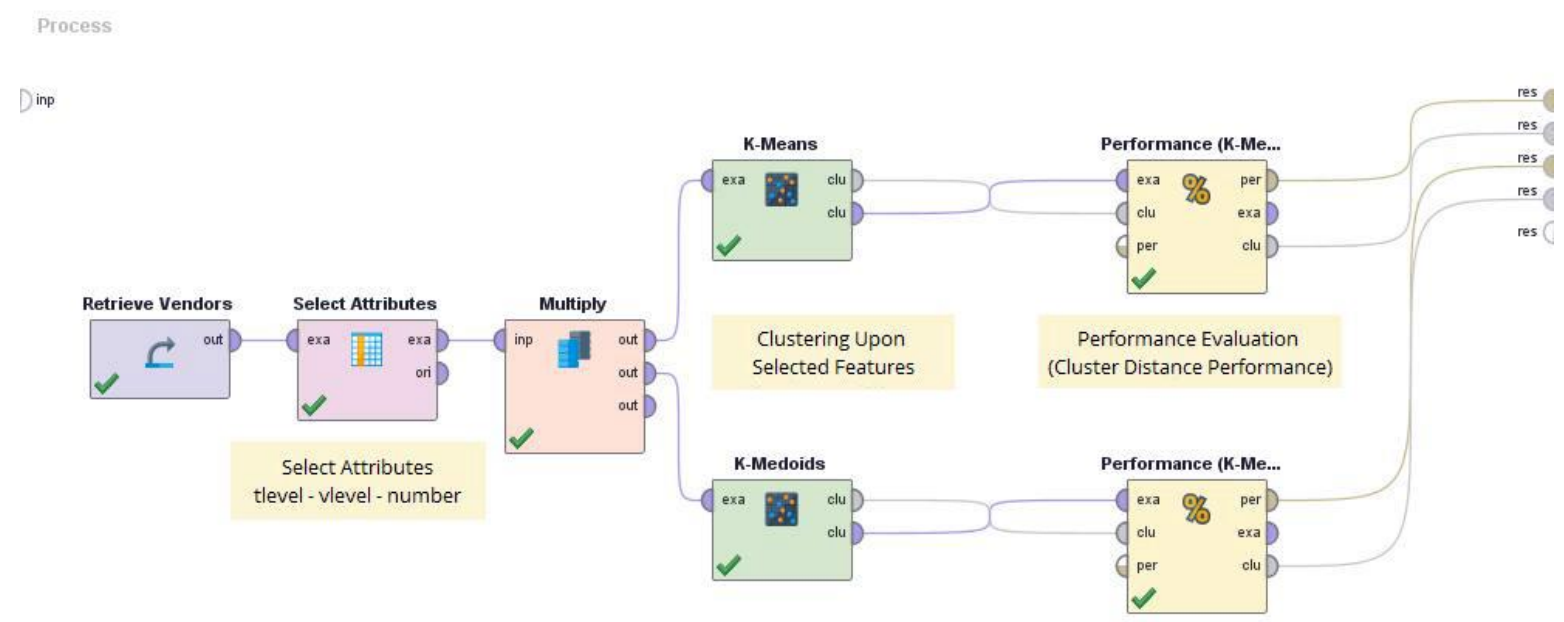

Fig.6. Process Design for Evaluating Performance of both K-Means and K-Medoids Algorithms in Vendors' Clustering

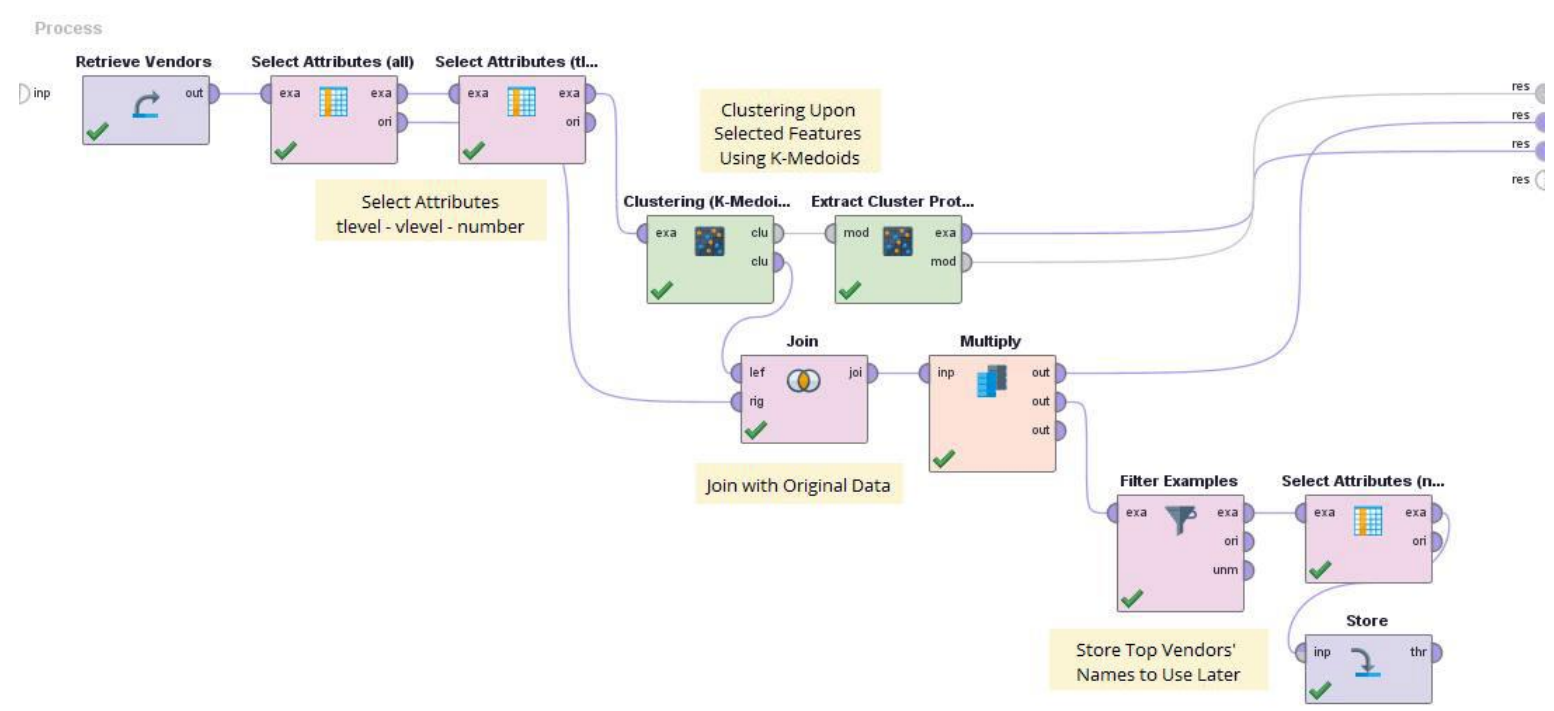

Fig.7. Process Design of Vendors Clustering Using K-Medoids Algorithm

Fig.8 illustrates the distribution of clusters elements in a 3D-Space created from the three selected attributes:

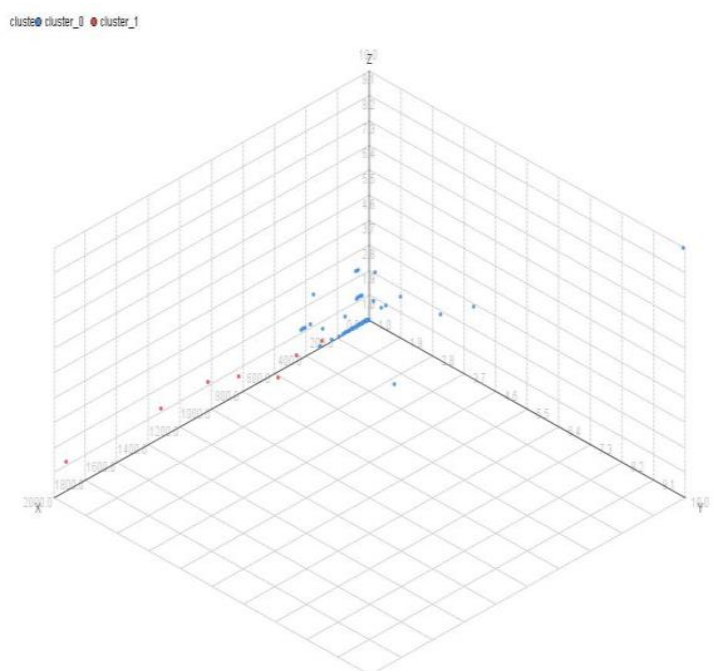

Fig.8. Vendors' Clusters Distribution in a 3D-Sapce
The extracted information may help in inferring that the mentioned vendors are the most professional, or their products are the most popular. It may also refer to the fact that they are companies or trade organizations due to their wide spreading products, as the latter (as we will see in the next section) form 1365 of 6387 items, i.e. $21.37 \%$ of the market stock.

\section{Clustering Products}

The available data about products are the following:

- Product title

- Vendor name

- Origin

- Shipment destination

- $\quad$ Price (in USD)

- Sales amount (since a specific date)

- Views number (since the same date)

- Since: the date when the mentioned sales and views had started

- Category 
In products clustering, we apply clustering concepts on two aspects as well: clustering products titles by considering them as documents and applying Documents Clustering, and the other is by taking advantage of the numerical attributes of the products.

In clustering products titles, we can divide the dataset by several ways, such as clustering products belonging to a particular category, or the products sold by specific vendors, or others. Here we found it is useful to cluster products sold by the Top Vendors, the approach that may introduce important information about the common characteristics among those vendors and among the products they promote.

In a similar way, we evaluated clustering performance with K-Means to choose the best possible number of clusters ${ }^{1}$.

Table 10 shows Davies-Bouldin Index values for different numbers of clusters:

Table 10. Davies-Bouldin Index Values for K-Means Algorithm in Products Titles Clustering According to Different Numbers of Clusters

\begin{tabular}{|c|c|}
\hline Number of Clusters (k) & Davies Bouldin \\
\hline 2 & 2.183 \\
\hline 3 & 2.309 \\
\hline 4 & 2.149 \\
\hline 5 & 2.212 \\
\hline 6 & 2.217 \\
\hline 7 & 3.439 \\
\hline
\end{tabular}

From this table, we notice approximate values, which clustering can depend on any. In our experiment, we chose the number 4, with Cosine Similarity for measuring.

Fig.9 illustrates the process design for clustering products titles of the Top Vendors. The process represents documents with vectors of words and their weights using TF-IDF. In additions, we use DF equals to 3 to remove extremely rare word appearing in less than 3 documents. This step forms a sub-process illustrated in Fig.10, and it consists of three operations: Tokenization, stemming with Porter Stemmer, and filtering tokens by length to filter words with length less than 2. These operations help in additional reduction of words amount and gaining more accurate clustering.

\section{i. Clustering Products Titles Results}

The above process produces the following distribution of elements upon clusters:

\begin{tabular}{l} 
Cluster Model: \\
Cluster 0: 612 items \\
Cluster 1: 323 items \\
Cluster 2: 209 items \\
Cluster 3: 221 items \\
Total number of items: 1365 \\
\hline
\end{tabular}

These elements represent vectors of words, which their count equals to 324 words, and their weights, and the centroids calculations are according to these weights. Table 11 shows the number of weighted words for each cluster (as the rest of the words have weights equal to 0 ), and Table 12 lists the first twenty words with the highest weights in each cluster:

Table 11. Number of Weighted Words in each Cluster

\begin{tabular}{|c|c|}
\hline Cluster & Number of Weighted Words \\
\hline cluster_0 & 289 \\
\hline cluster_1 & 52 \\
\hline cluster_2 & 67 \\
\hline cluster_3 & 155 \\
\hline
\end{tabular}

Table 12. The Top 20 Words with the Highest Weights in each Cluster

\begin{tabular}{|c|c|c|c|c|c|c|c|}
\hline \multicolumn{2}{|c|}{ cluster_0 } & \multicolumn{5}{c}{ cluster_1 } & \multicolumn{2}{c|}{ cluster_2 } & \multicolumn{2}{c|}{ cluster_3 } \\
\hline AttributeTF-IDF & Attribute & TF-IDF & Attribute & TF-IDF & Attribute TF-IDF \\
\hline crypter & 0.073 & databas & 0.396 & config & 0.360 & get & 0.278 \\
\hline make & 0.060 & record & 0.345 & mba & 0.246 & free & 0.266 \\
\hline crack & 0.058 & plaintext & 0.266 & sentri & 0.246 & card & 0.212 \\
\hline checker & 0.042 & com & 0.231 & snapshot & 0.243 & product & 0.076 \\
\hline brute & 0.039 & million & 0.119 & snipr & 0.240 & amazon & 0.040 \\
\hline monei & 0.032 & net & 0.044 & leagu & 0.036 & gift & 0.037 \\
\hline keylogg & 0.031 & buycraft & 0.039 & legend & 0.036 & samsung & 0.023 \\
\hline paypal & 0.030 & log & 0.037 & storm & 0.032 & iphon & 0.021 \\
\hline phish & 0.029 & forum & 0.032 & domino & 0.011 & video & 0.020 \\
\hline account & 0.027 & org & 0.019 & com & 0.011 & rdp & 0.020 \\
\hline page & 0.027 & account & 0.018 & uk & 0.009 & eat & 0.018 \\
\hline video & 0.025 & lifetim & 0.017 & cb & 0.008 & credit & 0.017 \\
\hline dai & 0.024 & porn & 0.017 & chaturb & 0.008 & drop & 0.016 \\
\hline pro & 0.022 & instant & 0.013 & crunchyrol & 0.008 & googl & 0.016 \\
\hline hack & 0.020 & deliveri & 0.013 & deezer & 0.008 & cloth & 0.015 \\
\hline cc & 0.018 & game & 0.009 & deviantart & 0.008 & laptop & 0.015 \\
\hline cvv & 0.018 & btc & 0.009 & expressvpn & 0.008 & method & 0.014 \\
\hline cashout & 0.018 & gamerzplanet & 0.008 & fitbit & 0.008 & game & 0.014 \\
\hline easi & 0.016 & ultim & 0.007 & gamestop & 0.008 & item & 0.014 \\
\hline ultim & 0.015 & minecraft & 0.006 & hulu & 0.008 & smart & 0.013 \\
\hline
\end{tabular}

In another perspective, and to take advantage of the numerical attributes of the products, we apply clustering on "views", "sales" and the date when the views and sales had started determined by "since". Here we calculate the duration in which the recorded views and sales took place, starting from "since" date until the date in which the crawler extracted the data from the website.

\footnotetext{
${ }^{1}$ We excluded applying K-Medoids algorithm due to the extreme time it consumes and without getting the desired results, therefore we settled for K-Means algorithm.
} 


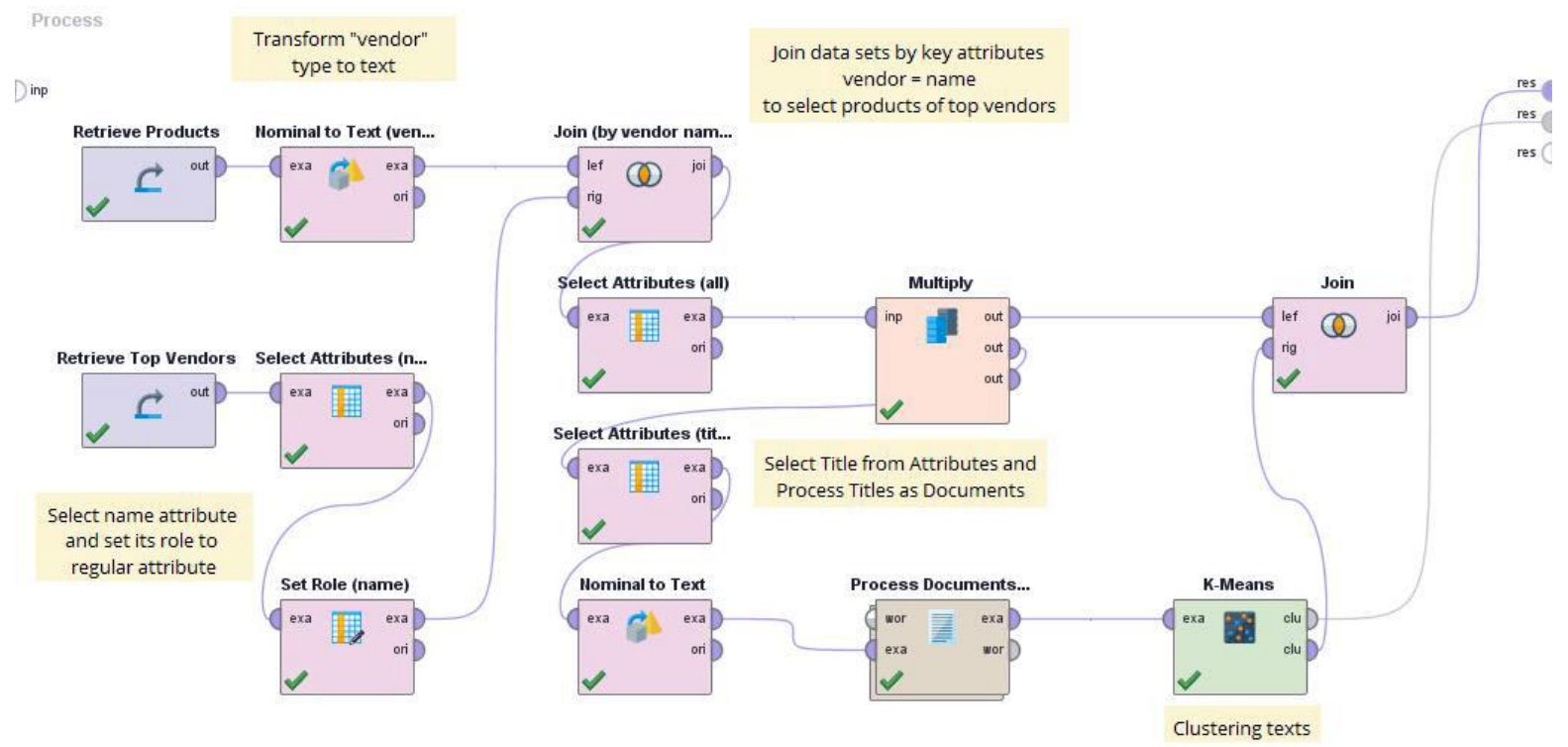

Fig.9. Process Design for Clustering Products Titles of the Top Vendors Using K-Means Algorithm

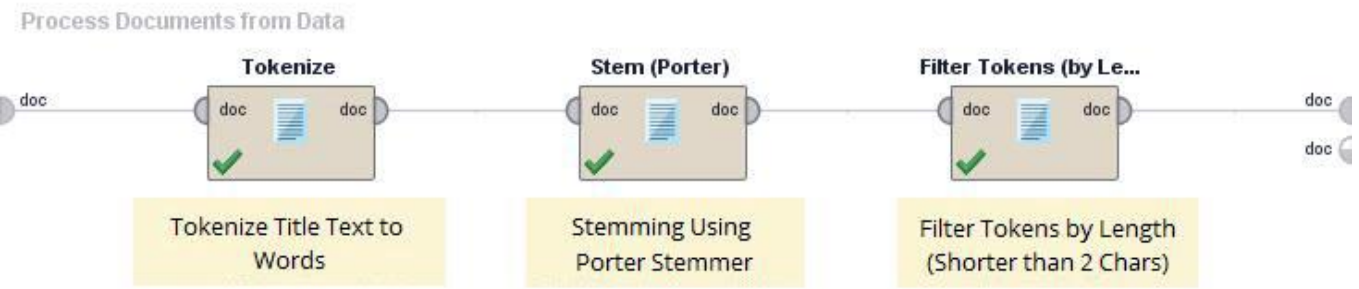

Fig.10. Processing Products Titles as Documents

Here also we found approximate values of DaviesBouldin Index as shown in Table 13, we chose the number 7, and the Euclidean Distance for measuring. Fig.11 illustrates the process design of products clustering according to views, sales and their durations.
Table 13. Davies-Bouldin Index Values in Evaluating Performance of Products Clustering using K-Means Algorithm

\begin{tabular}{|c|c|}
\hline Number of Clusters (k) & Davies Bouldin \\
\hline 2 & 0.505 \\
\hline 3 & 0.470 \\
\hline 4 & 0.528 \\
\hline 5 & 0.567 \\
\hline 6 & 0.552 \\
\hline 7 & 0.501 \\
\hline
\end{tabular}

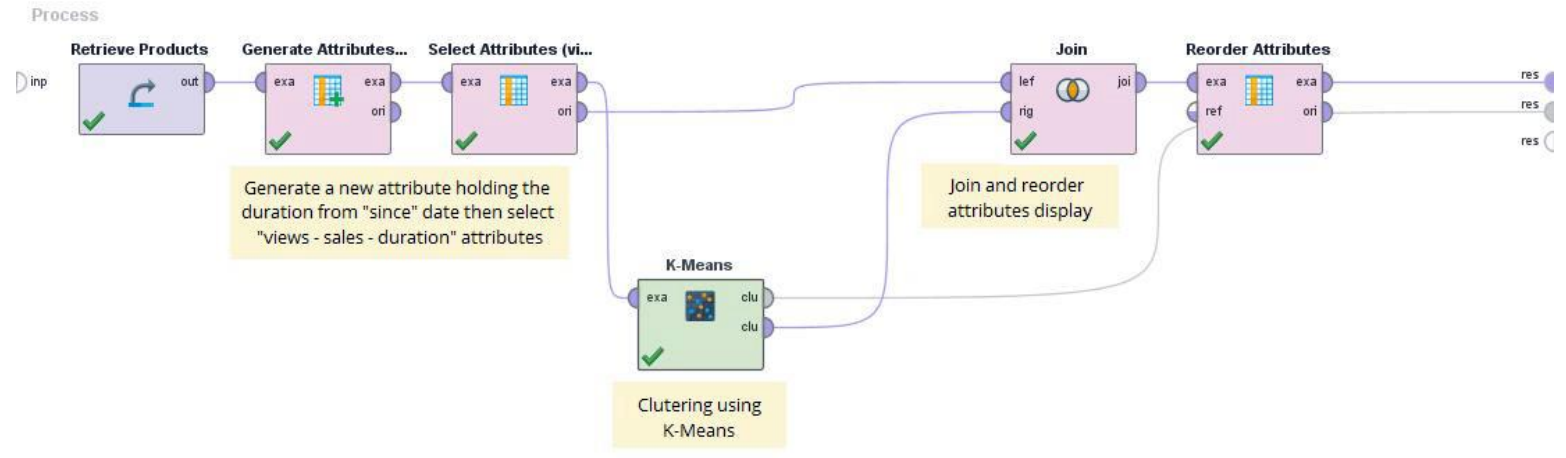

Fig.11. Process Design of Products Clustering According to View, Sales and their Occurring Durations 
The previous process produces the following

\section{ii. Clustering Products Results} distribution of the elements over clusters:

Table 14 lists the centroids values in the generated clusters, and Table 15 shows the elements in "cluster_2" and "cluster_3", which we notice that they include the products with the most views and sales:

Table 14. Centroids Values in the Generated Clusters of Products

\section{Cluster Model:}

Cluster 0: 143 items

Cluster 1: 2657 items

Cluster 2: 1 items

Cluster 3: 7 items

Cluster 4: 39 items

Cluster 5: 3055 items

Cluster 6: 485 items

Total number of items: 6387

\begin{tabular}{|c|c|c|c|c|c|c|c|}
\hline Attributecluster_0 & cluster_1 & cluster_2 & cluster_3 & cluster_4 & cluster_5 & cluster_6 \\
\hline sales & 33.909 & 0.849 & 367 & 267.714 & 76.872 & 0.204 & 7.381 \\
\hline views & 1206.86 & 196.526 & 13457 & 7446.4292780 .821 & 35.314 & 524.315 \\
\hline duration & 148.287 & 123.841 & 196 & 157.429 & 160.436 & 28.704 & 147.757 \\
\hline
\end{tabular}

Table 15. Items Details in "cluster_2" and "cluster_3"

\begin{tabular}{|c|c|c|c|c|c|c|c|c|c|c|}
\hline cluster & category & title & vendor & origin & shipto & price & views & sales & since & duration \\
\hline cluster_2 & Fraud & $\begin{array}{l}\text { super fresh high quality united } \\
\text { kingdom fullz }\end{array}$ & RangeRovers & $\begin{array}{l}\text { World } \\
\text { Wide }\end{array}$ & $\begin{array}{l}\text { World } \\
\text { Wide }\end{array}$ & 5 & 13457 & 367 & $\begin{array}{l}\text { Sun Mar } 04 \\
2018\end{array}$ & 196 \\
\hline cluster_3 & $\begin{array}{l}\text { Counterfeit } \\
\text { Items }\end{array}$ & $\begin{array}{l}\text { counterfeit } 20 \text { usd notes-high } \\
\text { quality-full escrow minimum } \\
\text { order } 5\end{array}$ & swimnotes & $\begin{array}{l}\text { United } \\
\text { States }\end{array}$ & $\begin{array}{l}\text { United } \\
\text { States }\end{array}$ & 3.99 & 8146 & 203 & $\begin{array}{l}\text { Sat Mar } 03 \\
2018\end{array}$ & 197 \\
\hline cluster_3 & Fraud & $\begin{array}{c}\text { usa hq cc } 100 \% \text { live known } \\
\text { balance } 1-5 \mathrm{k} 17\end{array}$ & TikTokCC & $\begin{array}{l}\text { World } \\
\text { Wide }\end{array}$ & $\begin{array}{l}\text { World } \\
\text { Wide }\end{array}$ & 17 & 9174 & 484 & $\begin{array}{c}\text { Fri May } 04 \\
2018\end{array}$ & 135 \\
\hline cluster_3 & Fraud & $\begin{array}{c}100 \text { us paypal accounts balance } \\
\text { paypal credit }\end{array}$ & Rambe & $\begin{array}{l}\text { World } \\
\text { Wide }\end{array}$ & $\begin{array}{l}\text { World } \\
\text { Wide }\end{array}$ & 6 & 6655 & 128 & $\begin{array}{c}\text { Fri Mar } 16 \\
2018\end{array}$ & 184 \\
\hline cluster_3 & Fraud & $\begin{array}{l}\text { cvv usa high quality best quality } \\
100 \% \text { live known balance } 10005 \mathrm{k}\end{array}$ & savastano & $\begin{array}{l}\text { World } \\
\text { Wide }\end{array}$ & $\begin{array}{l}\text { World } \\
\text { Wide }\end{array}$ & 15 & 5444 & 277 & $\begin{array}{l}\text { Mon Apr } \\
162018\end{array}$ & 153 \\
\hline cluster_3 & Fraud & $\begin{array}{l}\text { live usa cvv known balance } 1 \mathrm{k} 5 \mathrm{k} \\
5 \mathrm{k} 20 \mathrm{k} 20 \mathrm{k} 50 \mathrm{k} \text { buy } 2 \text { get } 1 \text { free }\end{array}$ & friskmint & $\begin{array}{l}\text { World } \\
\text { Wide }\end{array}$ & $\begin{array}{l}\text { World } \\
\text { Wide }\end{array}$ & 10 & 7679 & 336 & $\begin{array}{l}\text { Sun Jul } 08 \\
2018\end{array}$ & 70 \\
\hline cluster_3 & Fraud & $\begin{array}{l}\text { usa cvv best quality market hq live } \\
100 \% \text { known balance } 5 \mathrm{k} 15 \mathrm{k}\end{array}$ & savastano & $\begin{array}{l}\text { World } \\
\text { Wide }\end{array}$ & $\begin{array}{l}\text { World } \\
\text { Wide }\end{array}$ & 18 & 9288 & 407 & $\begin{array}{c}\text { Tue Apr } 17 \\
2018\end{array}$ & 152 \\
\hline cluster_3 & $\begin{array}{l}\text { Guides \& } \\
\text { Tutorials }\end{array}$ & $\begin{array}{l}\text { video proofs wanna make } 77400- \\
158000 \text { day }\end{array}$ & MrMillionaire & $\begin{array}{l}\text { World } \\
\text { Wide }\end{array}$ & $\begin{array}{l}\text { World } \\
\text { Wide }\end{array}$ & 499 & 5739 & 39 & $\begin{array}{l}\text { Sat Feb } 17 \\
2018\end{array}$ & 211 \\
\hline
\end{tabular}

\section{CONCLUSION AND FUTURE WORK}

In this paper, we discussed how to infer useful information from data extracted from a dark web market. This information helps security and law agencies in their investigations about activities that take place on the dark web.

We introduced our approach of a system that consists of three modules. The first module is The Dark Crawler Darky, which accesses a dark web market, by simulating a user login to the market, scans the whole website, and extracts and directly structures the extracted data. The second module is The Cleaner, which applies preprocessing procedures on extracted data, including cleaning and transformation, to prepare data for mining. The third and final module is The Dark Miner, which consists of mining processes in the fields of Association Rules and Clustering, illustrating all gained results. We applied text mining on products titles by considering each title as a document. In the field of Association Rules, we extracted frequent words used by vendors when they promote their products, and from the generated rules we inferred conceptual compositions used in titling products. In the field of Clustering, we employed Clustering techniques to find a relationship among some specific attributes in the vendors' data, which are the Vendor Level, Trust Level, and the bracketed (mysterious) Number. The process clusters vendors according to these three attributes, and inferring that they might have a correlation, leading to the Top Vendors (or vendors with the most professionalism and interactivity). Next, we applied clustering on products titles of the Top Vendors to find the common characteristics they share according to the products they promote, achieving this through the highest weighted terms. Lastly, we performed clustering on products according to views, sales and the duration of when the mentioned views and sales have occurred.

This approach can apply several data mining techniques on extracted data. The design of the crawler differs from a dark website to another, as well as data mining operations may differ according to the website nature and quality of the data contained in the website. And for the same website, results will vary from a period 
of time to another, as products may significantly increase with various promotion styles, in addition to increase in vendors' number. This approach puts a dark website under investigation and in any time.

In its future prospects, we develop the system to cover other methods of data mining, such as Classification integrated with Clustering, by using the generated clusters as labels, or integrated with Association Rules in a methodology of Associative Classification. Also we expand our experiments to include markets in other languages, and involve techniques in the field of preprocessing, including corrections of the misspelled words and words containing purposely-altered characters with symbols or letters from mixed languages.

\section{REFERENCES}

[1] B. Hawkins, "Under The Ocean of the Internet - The Deep Web," $15 \quad 5$ 2016. [Online]. Available: https://www.sans.org/reading-

room/whitepapers/covert/ocean-internet-deep-web-37012. [Accessed 13 December 2018].

[2] M. S. L. S. W. L. Andres Baravalle, "Mining the Dark Web: Drugs and Fake Ids," in Data Mining Workshops (ICDMW), 2016 IEEE 16th International Conference, Barcelona, Spain, 2016.

[3] J. A. F. A. R. W. Monica J. Barratt, "Safer Scoring? Cryptomarkets, Social Supply and Drug Market Violence," International Journal of Drug Policy, vol. 35, pp. 24-31, 2016.

[4] A. D. ,. E. M. ,. E. N. ,. V. P. ,. J. S. ,. P. S. John Robertson, Darkweb Cyber Threat Intelligence Mining, Cambridge: Cambridge University Press, 2017.

[5] O. B. M. N. S. Quan Le, "Deep Learning at the Shallow End: Malware Classification for Non-Domain Experts," Digital Investigation, vol. 26, pp. S118-S126, 2018.

[6] G. M. M. M. Alessandro Celestini, "Tor Marketplaces Exploratory Data Analysis: The Drugs Case," in International Conference on Global Security, Safety, and Sustainability, 2017.

[7] M. A. E. N. P. S. Ericsson Marin, "Community Finding of Malware and Exploit Vendors on Darkweb Marketplaces," in In 2018 1st International Conference on Data Intelligence and Security (ICDIS), 2018.

[8] F. C. B. B. Paolo Spagnoletti, "An Investigation on the Generative Mechanisms of Dark Net Markets," in Proceedings of the 13th Pre-ICIS Workshop on Information Security and Privacy, 2018.

[9] D. L. N. A. S. A. M. P. M. S. Ben R. Lane, "The Dark Side Of The Net: Event Analysis Of Systemic Teamwork (East) Applied To Illicit Trading On A Darknet Market," in Proceedings of the Human Factors and Ergonomics Society 2018 Annual Meeting, 2018.

[10] I. Ladegaard, "Crime Displacement in Digital Drug Markets," International Journal of Drug Policy, vol. 63, p. 113-121, 2019.

[11] D. R. M. M. L. S. Q. R. Julian Broséus, "A Geographical Analysis of Trafficking on a Popular Darknet Market," Forensic science international, vol. 277, pp. 88-102, 2017.

[12] H. P. Petar Ristoski, "Semantic Web in data mining and knowledge discovery: A comprehensive survey," Web Semantics: Science, Services and Agents on the World Wide Web, vol. 36, pp. 1-22, 2016.

[13] P. R. A. S. P. S. Praveen Kumari, "Web Mining - Concept, Classification and Major Research Issues: A Review,"
Asian J. Adv. Basic Sci, vol. 4, no. 2, pp. 41-44, 11 May 2016.

[14] L. H. P. P. C. Pritam C. Gaigole, "Preprocessing Techniques in Text Categorization," in National Conference on Innovative Paradigms in Engineering \& Technology (NCIPET-2013), 2013.

[15] J. I. N. S. Vijayarani, "Preprocessing Techniques for Text Mining - An Overview," International Journal of Computer Science \& Communication Networks, vol. 5, no. 1, pp. 7-16, 2015.

[16] S. K. I. A. K. M. A. A. Rida Hafeez, "Does Preprocessing Really Impact Automatically Generated Taxonomy," in 2017 13th International Conference on Emerging Technologies (ICET), 2017.

[17] J. T. P. Surbhi K. Solanki, "A Survey on Association Rule Mining," in 2015 Fifth International Conference on Advanced Computing \& Communication Technologies, 2015.

[18] J. T. P. Surbhi K. Solanki, "A Survey on Association Rule Mining," in 2015 Fifth International Conference on Advanced Computing \& Communication Technologies, 2015.

[19] T. Vishal, "Cluster Analysis for Market Segmentation," 3 February 2015. [Online]. Available: https://www.slideshare.net/vishtandel1991/clusteranalysis-for-market-segmentation. [Accessed 9 March 2019].

[20] N. P. M. P. Jasmine Irani, "Clustering Techniques and the Similarity Measures used in Clustering: A Survey," International Journal of Computer Applications, vol. 134, no. 7, pp. 9-14, January 2016.

[21] R. B. Bassel AlKhatib, "Crawling the Dark Web: A Conceptual Perspective, Challenges and Implementation," Journal of Digital Information Management (JDIM), vol. 17, no. 2, pp. 51-60, April 2019.

[22] "Davies-Bouldin Criterion Clustering Evaluation Object MATLAB - MathWorks Benelux," [Online]. Available: https://nl.mathworks.com/help/stats/clustering.evaluation. daviesbouldinevaluation-class.html. [Accessed 19 April 2019].

\section{Authors' Profiles}

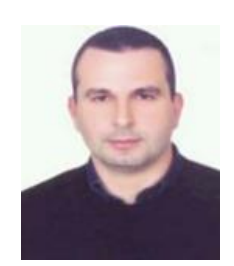

Bassel Alkhatib is the web sciences master director at the Syrian Virtual University and the head of Artificial Intelligence department at Information Technology Faculty at Damascus University. He holds PhD degree in computer science from the University of Bordeaux-France, 1993. Dr. Alkhatib supervises many $\mathrm{PhD}$ students in web mining, and knowledge management. He also leads and teaches modules at both BSc and MSc levels in computer science and web engineering in Syrian Virtual University, Damascus University, and Al-Shem Private University.

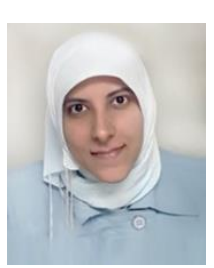

Randa S. Basheer is a web sciences master student at Syrian Virtual University. She has a Bachelor degree in information technology engineering, Damascus University, Damascus, Syria, 2006. Ms. Basheer coauthored a published article with Dr. Alkhatib: "Crawling the Dark Web: A Conceptual Perspective, Challenges and 
Implementation", Journal of Digital Information Management Web. (JDIM), April 2019. Her current research of interest is the Dark

How to cite this paper: Bassel Alkhatib, Randa S. Basheer, " Mining the Dark Web: A Novel Approach for Placing a Dark Website under Investigation", International Journal of Modern Education and Computer Science(IJMECS), Vol.11, No.10, pp. 1-13, 2019.DOI: 10.5815/ijmecs.2019.10.01 